\title{
EXPERIMENTACIÓN Y REPRESENTACIÓN EN LA NOVELA COLOMBIANA ACTUAL: JUAN Cárdenas, Margarita García Robayo y JUAN Álvarez*
}

\author{
EXPERIMENTATION AND Representation \\ in the Contemporary Colombian Novel: \\ Juan Cárdenas, Margarita García \\ Robayo and Juan Álvarez
}

\footnotetext{
* Una primera versión de este texto fue leída como conferencia en el evento "Narrar en Colombia y en Argentina hoy", realizado el 12 de noviembre de 2019 en la Universidad de Granada, en Granada, España.
}

Cómo citar este artículo: Torres Perdigón, A. (2021). Experimentación y representación en la novela colombiana actual: Juan Cárdenas, Margarita García Robayo y Juan Álvarez. Estudios de Literatura Colombiana 48, pp. 135-152. DOI: https://doi. org/10.17533/udea.elc.n48a09

1 https://orcid.org/0000-0003-2539-4766 andreatorrespa@gmail.com Pontificia Universidad Javeriana, Colombia

Editores: Andrés Vergara Aguirre, Christian Benavides Martínez, Valentina Noreña Gómez

Recibido: 13.08 .2020

Aprobado: 26.10.2020

Publicado: 18.12 .2020

Copyright: (2021 Estudios de Literatura Colombiana. Este es un artículo de acceso abierto distribuido bajo los términos de la Licencia Creative Commons AtribuciónNo comercial - Compartir igual 4.0 Internacional

\section{Andrea Torres Perdigón ${ }^{1}$}

Resumen: Este artículo analiza tres textos de autores colombianos contemporáneos: Los estratos, Lo que no aprendi y La ruidosa marcha de los mudos, de Juan Cárdenas, Margarita García Robayo y Juan Álvarez, respectivamente. Por medio del análisis de características de las novelas, el artículo explora la relación entre rasgos de lo que llamamos representación, en el sentido de unos referentes sociales e históricos reconocibles, $\mathrm{y}$ otros de lo que identificamos como experimentación en procedimientos formales singulares de cada novela. Proponemos que esta tensión permite pensar de otras maneras los discursos sobre esos mismos referentes reconocibles de la historia y del contexto colombiano contemporáneo.

Palabras clave: Juan Cárdenas; Margarita García Robayo; Juan Álvarez; novela colombiana; política de la literatura.

\begin{abstract}
This article analyses three novels of Colombian contemporary authors: Juan Cárdenas, Margarita García Robayo and Juan Álvarez. Through the analysis of some characteristics of the novels Los estratos, Lo que no aprendi and La ruidosa marcha de los mudos, the article explores the link between what we call representation, in the sense of some recognizable social and historical referents, and what we identify as experimentation, e. g. formal and singular procedures of each novel. We suggest that this tension allows us to think in a different way discourses about those same recognizable referents of Colombian history and contemporary context.
\end{abstract}

Keywords: Juan Cárdenas; Margarita García Robayo; Juan Álvarez; Colombian novel; politics of literature. 
Siete mil hombres en tres meses, [sic] depusieron odio, zanjaron cuentas con la guerra, el hambre y la justicia, y saliendo de madrigueras, cuevas y refugios dieron acto de fe: aquí tienen las armas, ya no peleamos más, fue la promesa contra lo prometido del gobierno; que quién sabe por qué ordenó una masacre campesina año y medio más tarde, en el Tolima, y en el cincuenta y cinco declaró Zona de operaciones militares a Villarrica, Carmen de Apicalá, Icononzo, Cunday, Pandi, Cabrera y a todo el Sumapaz, y comenzó a invadir, a asesinar, a destazar, violar, a torturar, a provocar presiones, desalojar a la gente de su tierra, incendiar, bombardear Albalucía Ángel (1975, p. 301).

\section{Apuntes sobre la tradición colombiana}

La historia de Colombia en el siglo xx y lo que llevamos del xxi está profundamente permeada por un conflicto armado interno prolongado, por acontecimientos múltiples y complejos de una violencia muy fuerte y a la vez heterogénea (tanto en sus formas como en sus actores y víctimas), y también por una desigualdad social exacerbada. Estas características, además de otra serie de aristas complejas de la historia colombiana, no han sido ajenas a la producción de los textos literarios. La tradición narrativa colombiana, en especial del siglo xx y xxi, contempla autores y textos que se han relacionado con esa historia de maneras diversas. Veamos brevemente algunas de ellas.

Por un lado, tenemos autores que dialogan intensamente con esas condiciones históricas al usarlas como referente de las ficciones o de lo que se ha llamado "novela testimonial" (testimonio sobre las violencias, sobre el conflicto armado, pero también sobre pugnas políticas y sociales de varias épocas). Por ejemplo, la autora de nuestro epígrafe, Albalucía Ángel (1939-), ha sido leída en esa clave, específicamente con respecto a lo que se conoce como época de La Violencia (1946-1958) en su novela Estaba la pájara pinta sentada en el verde limón (1975). Por otro lado, tenemos a quienes poco mencionan esas condiciones en términos referenciales (es decir, que sus textos casi no aluden a estas situaciones o a los distintos acontecimientos históricos nacionales dentro de sus temas). R. H. Moreno-Durán (1946-2005) podría fungir de ejemplo de este tipo de narrativa, aunque también algunos textos de Pablo Montoya (1963-) como Tríptico de la infamia. Pues bien, este artículo busca una tercera forma de relación entre los textos narrativos y la historia colombiana. Por fuera del ámbito del testimonio o de una escritura referencial de la historia, y más allá de la constatación de la ausencia de temas históricos y políticos nacionales en ciertos autores, buscamos acercarnos a la narrativa colombiana contemporánea a partir de una tensión productiva entre la representación del mundo histórico y social, por un lado, y la experimentación con 
las formas literarias, por otro. Aunque todo el texto girará en torno a estos dos puntos, de manera esquemática podemos anunciar que por representación entendemos unos referentes sociales e históricos que son reconocibles en los textos, y con experimentación nos referimos a procedimientos formales singulares de cada novela. Si se quiere, la representación también tiene que ver aquí con la afirmación clásica de Erich Auerbach (1950) en la que "la interpretación de lo real por la representación literaria o “imitación”" (p. 522) se vincula con varias formas del problema del realismo. Como lo explica Marina Mackay (2011), "Three main features constitute realism here: the mixing of styles, from the high literary style to ordinary demotic language; the serious treatment of the everyday lives of the ordinary, un-aristocratic masses; the embedding of those ordinary lives in their very specific social and historical contexts" (p. 12). En este caso, los dos últimos rasgos tienen que ver con lo que identificamos como representación también: la alusión a la vida cotidiana de las masas y el hecho de enmarcarlas en contextos sociales e históricos específicos.

En esta medida, la relación entre la historia colombiana y los textos literarios será explorada a partir de novelas contemporáneas que, como procuraré argumentar, experimentan con las formas para repensar esa historia o para imaginar distintos presentes. Esto no implica que el contexto histórico colombiano emerja como el único tema central de estos textos contemporáneos, sino que estos proponen unas formas distintas al mero código referencial para pensar y leer la historia colombiana y nuestro presente globalizado. Proponemos primero dos aclaraciones sobre el problema entre la experimentación y la representación, para luego entrar en la exposición de tres textos colombianos contemporáneos y, por último, al análisis de algunas de sus formas para representar y experimentar. El propósito es proponer una lectura de esos dos polos de la producción literaria a través del análisis de procedimientos formales concretos de las tres novelas, a partir de un enfoque inspirado en los procedimientos propuestos por Tynianov (1969), como se aclarará más adelante.

\section{¿Por qué hablar de experimentación y representación?}

El hecho de tener una historia violenta y confusa no es una característica exclusiva de la tradición colombiana: es, más bien, uno de los rasgos que, además del pasado colonial, comparten varios de los países hispanoamericanos o incluso del sur global, aunque con singularidades marcadas. En esta medida, ¿no podrían todas las literaturas nacionales 
leerse desde esta tensión entre experimentación formal y representación histórica o social? Quizá sí, por lo menos en cierto tipo de textos que exploran dimensiones creativas frente al canon o que son poco repetitivas de fórmulas narrativas fácilmente comercializables. Sin embargo, hay tres aclaraciones que cabe hacer vinculadas al caso colombiano y que le darían pertinencia a esta forma de leer esta narrativa contemporánea.

La primera aclaración consiste en que hay una tradición de textos narrativos que han experimentado con las formas e insistido en contenidos históricos o situaciones sociales concretas. Textos canónicos como La vorágine (1924), de José Eustasio Rivera, o El otoño del patriarca (1975), de Gabriel García Márquez, manifiestan ese trabajo simultáneo entre las formas experimentales en la escritura y la búsqueda de una reflexión — más que la mera representación — sobre el genocidio cauchero a comienzos del siglo xx en la Amazonia, en el caso de Rivera, y sobre la figura del dictador latinoamericano y su soledad en el poder, en el caso de García Márquez. La misma Albalucía Ángel (1975) puede ser leída desde esa tensión entre experimentación y representación, puesto que se mueve entre el registro infantil e íntimo de su personaje principal y el registro casi periodístico en la narración del Bogotazo, de las masacres posteriores y del surgimiento de las guerrillas. También es notorio el uso frecuente del discurso indirecto libre con marcas diatópicas explícitas, además de un manejo temporal que busca imitar la configuración errática de los recuerdos de la infancia, en lugar de una reconstrucción aparentemente objetiva de la historia colombiana desde 1948 hasta los años setenta. Estos ejemplos son muestra de una tradición narrativa colombiana que ha elaborado esa tensión entre la experimentación formal y la representación del mundo social o histórico, tensión que en cada caso ha permitido la creación de novelas muy diversas. Esta tradición implica que el problema que tratamos aquí no es nuevo ni ha emergido en el vacío, sino que conversa con textos del canon y con las distintas maneras en las que se ha pensado dentro de la narrativa colombiana.

La segunda aclaración es que la historia colombiana sigue en disputa. En esta medida, se trata de una historia muy vigente, puesto que aún hay actores que se disputan la realidad de ciertos acontecimientos o que buscan ocultar lo que se sabe acerca de otros. Esto implica que no se está ante una historia ya analizada y digerida a posteriori, con un proceso colectivo de creación de memoria histórica, sino ante un forcejeo en torno a cómo se cuenta esa historia y quiénes la cuentan. Y esto ocurre con procesos desde la Independencia hasta la historia más reciente del conflicto ar- 
mado que trató de reconstruir el Grupo de Memoria Histórica, al menos hasta antes de la llegada en 2018 del gobierno de ultraderecha actual. Además de esa disputa por la historia, hay condiciones sociales de desigualdad que son actuales y que afectan la vida cotidiana de los colombianos hoy (más aún en el contexto de la pandemia por Covid-19). Así, las condiciones sociales están presentes y visibles en las discusiones públicas y académicas en la sociedad colombiana contemporánea, aspecto que también explica por qué la representación en la novela contemporánea es un tema relevante para los estudios sobre literatura colombiana.

La última aclaración tiene que ver con por qué hablar de experimentación y representación, pero ya no solo desde el contexto colombiano. En este caso, nos parece que las reflexiones teóricas sobre la literatura pasan por un momento particularmente referencial, es decir, en el que se privilegian los referentes culturales e históricos. Recientemente, y después del descrédito de movimientos posestructuralistas y su fuerte vínculo con el lenguaje y con el giro lingüístico, ${ }^{1}$ parece que estuviéramos ante un reclamo por la vuelta a lecturas referenciales de los textos: el dominio de textos hipernarrativos (como si fuesen más referenciales que otros), el auge de los textos autobiográficos y de su estudio, ${ }^{2}$ la reivindicación de la no ficción, ${ }^{3}$ entre otras manifestaciones, podrían dar cuenta de esta orientación. ${ }^{4}$ Desde el interés por los objetos y las presencias — como en el caso de Gumbrecht (2004) — ${ }^{5}$ hasta la fuerte influencia de los estudios poscoloniales y decoloniales, al menos en la academia latinoamerica-

Ese descrédito se observa en el abandono del abordaje de los textos en términos de lenguaje o estéticos, a favor de análisis que reivindican más los espacios sociales, identitarios y culturales. Como lo señala Eduardo Becerra Grande (2015): "las nuevas discusiones parecen apuntar sobre todo a problemas situados más allá del terreno estrictamente estético: como la función de la literatura dentro de la textura cultural del presente o incluso su validez como categoría o disciplina a la hora de analizar el espacio social y de actuar dentro de él" (párr. 1).

Como lo muestra el estudio de Julia Érika Negrete Sandoval (2015), quien busca no tanto estudiar la autobiografía, sino "sopesar su estado tanto en la producción literaria hispanoamericana de las décadas de 1960 y 1970, como en el marco de las teorías que se gestaron por los mismos años y que propiciaron la creación de la autoficción, modalidad genérica que marcó el impase en los estudios autobiográficos actuales" (p. 222).

Piénsese en el caso de autores como Héctor Abad Faciolince, en el caso colombiano.

$4 \quad$ Cuando hablamos de textos hipernarrativos nos referimos a novelas en las que prima la anécdota —e incluso la proliferación de anécdotas y eventos-, por encima de otros rasgos menos lineales. En el escenario colombiano, podemos dar el ejemplo de autores como Juan Gabriel Vázquez.

5 De manera resumida, Gumbrecht (2004) propone superar el signo como elemento de reflexión para las humanidades en lo que llama el estudio de efectos de la presencia: "Now what would it mean —and what would it take - to put an end to the age of the sign? What would it mean — and what would it take — to end metaphysics? It can certainly not mean that we abandon meaning, signification, and interpretation. [...] it would mean to try and develop concepts that could allow us, in the Humanities, to relate to the world in a way more complex that interpretation alone, that is more complex that only attributing meaning to the world" (p. 52). 
na, estamos en un momento que trabaja los textos literarios desde su referencialidad frente a contextos o situaciones específicas: en esta medida, en ocasiones se contempla como un objeto de estudio sin mediaciones, es decir, como si los textos trataran problemas históricos como una comunicación directa y transparente. El hecho de emplear contextos históricos o referenciales para la narrativa parece un valor literario en la actualidad, así como representar gestos de resistencia social o política, o recrear voces que históricamente han sido silenciadas, en sintonía con ciertos paradigmas contemporáneos de las ciencias sociales. En este contexto, es importante poner en diálogo esos valores de lo referencial, que aquí llamaremos representación de lo social o de lo histórico, con una noción tal vez benjaminiana de la forma, es decir, con las transformaciones y experimentaciones formales que ocurren en ciertos textos y que determinan una relación particular entre lo político y lo literario, o más bien, una política literaria, como la ha planteado Jacques Rancière (2010).

Benjamin (2009) propone una forma en la que siempre está presente la relación con la producción y con el hecho de que "el tratamiento dialéctico de los varios problemas literarios, [...] no sabe qué hacer con las cosas aisladas: obra, novela o libro. Tiene que integrar a cada cosa en conexiones sociales que estén vivas" (p. 299). De esa manera, los textos entendidos como producciones están siempre vinculados con sus contextos, pero su modo de intervención está en la técnica, concepto que "representa el punto de partida dialéctico para así superar la contraposición estéril que se da entre forma y contenido" (p. 300). La técnica literaria permitiría pensar en otras maneras de relación entre lo político y lo literario.

Por su parte, Rancière (2010) plantea su propuesta de una política literaria que se da por medio de los propios rasgos y formas literarias, y no en sus contenidos referenciales (sean políticos o históricos) o en las posturas de los escritores. Es en sus modos de escritura y en sus formas que la literatura tiene una política propia: “La expresión 'política de la literatura' implica entonces, que la literatura interviene en tanto que literatura en ese recorte de los espacios y los tiempos, de lo visible y lo invisible, de la palabra y el ruido" (p.16). Los textos literarios, a través de sus formas, intervienen en ese reparto de lo común.

Ante un giro teórico y crítico hacia lo referencial y lo no mediado, es decir de análisis que no se centran propiamente en los textos, sino en sus temas y en la relación de estos con problemas sociales y culturales, queremos explorar la tensión entre la 
representación y la experimentación, es decir, entre la referencialidad histórica y social y su diálogo tenso con la mediación de las formas literarias. Entendemos aquí la experimentación ligada a lo que ya los formalistas rusos identificaban con los procedimientos, en el sentido de una característica del estilo o una forma de escritura dada que se puede mecanizar y, por ende, reproducir posteriormente. Tynianov estudia estos procedimientos de manera especial en la parodia:

La esencia de la parodia reside en la mecanización de un procedimiento definido, mecanización perceptible solo en el caso en el que, evidentemente, el procedimiento sobre el cual se ejerce es conocido. Así la parodia cumple un doble objetivo: I) mecaniza un procedimiento definido, 2) organiza una materia nueva que no es otra que el antiguo procedimiento mecanizado (Tynianov, 1969, p. 74, traducción propia).

Hay procedimientos que se pueden mecanizar - y en ese caso producen la parodiaDe ahí tomaremos la idea del procedimiento como una forma que puede o no producir mecanizaciones posteriores en otros autores, pero que es una forma reconocible en los textos.

Esta exploración la proponemos en tres autores contemporáneos: Juan Cárdenas (1978-), Margarita García Robayo (1980-) y Juan Álvarez (1978-). En particular, abordaremos una novela en específico de cada uno de ellos: Los estratos (2013), Lo que no aprendí (2014) y La ruidosa marcha de los mudos (2015), respectivamente. Se trata de textos de reciente publicación, y en esa contemporaneidad reside parte de su interés; sin embargo, además de ser contemporáneos, en estas tres novelas se trabaja el problema de las formas literarias y, a la vez, se piensa en las configuraciones de lo histórico y literario en el contexto colombiano. A continuación, explicaremos de qué maneras.

\section{Presentación de Los estratos, Lo que no aprendí y La ruidosa marcha de los mudos} En Los estratos tenemos a un personaje principal que pertenece a una clase social acomodada en la ciudad de Cali. De hecho, este personaje hereda una fábrica de gelatina de su padre, aunque la empresa entra en quiebra a medida que avanza la novela. La vida aparentemente estable y acomodada de este personaje clínicamente depresivo se va desarticulando poco a poco, a medida que inicia la búsqueda de una nana que desapareció abruptamente en su infancia. Mientras se ocupa de la reconstrucción de ese recuerdo que lo persigue y de la búsqueda de su nana, la empresa quiebra, se separa de su esposa, y todo empieza a girar solamente en torno a su bús- 
queda. Esta implica, en una estructura parcialmente análoga a la de La vorágine, un viaje hacia el puerto en el que recuerda una escena con la nana, y el posterior ingreso a la selva con la guía de un supuesto detective que es indio, para llegar al encuentro con una comunidad de negros y la identificación con ellos. Aunque sabemos que se desarrolla entre Cali y Buenaventura, por menciones ocasionales al puerto y al paso de la cordillera occidental, el narrador principal no menciona los nombres propios que tienen que ver con el mundo narrado de la novela: ni de personajes ni de lugares. Como lo dirá el mismo narrador y personaje, "Tal como me cuesta a mí pronunciarlo, mi nombre y todos los demás nombres. No puedo decir nombres. Algo no me deja" (Cárdenas, 2013, p. 127). Parte del trabajo de Cárdenas aquí tiene que ver con ese uso de los nombres comunes para referirse a todos los elementos de la narración. En una entrevista con María Paulina Ortiz (2014), Cárdenas aclara esa elección y la de la mención explícita a La vorágine: "Pensé: qué pasa si no incluyo nombres propios, pero pongo uno solo. Sobre todo quería dejar clara la alusión a ese libro que me parece importantísimo, increíble y que, si te fijas bien, es otro de esos grandes libros sobre el idioma, sobre la lengua" (párr. 14). De ahí queda reiterado el papel relevante del clásico de José Eustasio Rivera en Los estratos, y la conciencia en torno al uso de nombres comunes y a la omisión de nombres propios. La psiquiatra, el indio, la comunidad de negros, la nana, la esposa, la modernita: estos son algunos ejemplos de esa nominalización. De esta manera, una lectura exclusivamente individualizante o subjetivista de una búsqueda "personal" en esta novela quizá sea poco pertinente, como lo explicaremos más adelante.

Por otro lado, el título alude a tres dimensiones de los estratos: la geológica, que aparece en las tres partes de la novela ("Falla", "Sedimento", "Temblor") como un movimiento por distintas capas; la subjetiva, a medida que el personaje inicial se va transformando y reconstruyendo por medio de la búsqueda de su recuerdo y de su encuentro con las poblaciones negras que termina en una toma de remedio (o de yagé); y la que alude a las clases sociales: es común en Colombia usar una acepción de la palabra "estratos" para referirse a las condiciones socioeconómicas de las personas (de hecho, y por inverosímil que parezca para ciudadanos de otros países, las ciudades tienen números que determinan el estrato socioecónomico: hay barrios estrato uno, dos, tres, cuatro, cinco y seis). En esta medida, coincidimos con Catalina Quesada (2017) cuando describe la estructura de la novela: 
Compuesta de tres partes — "Falla", "Sedimento" y "Temblor"-, la novela Los estratos recurre a la metáfora geológica para explorar los entresijos de la sociedad colombiana contemporánea a distintos niveles, a la vez que el narrador indaga en los estratos de su propia conciencia, sus recuerdos y su pasado (p. I68).

Con respecto a esta interpretación, solo añadiremos que esa metáfora geológica implica que tanto la dimensión subjetiva como la social se exploran como estructuras que se van reconfigurando a medida que el narrador las va atravesando casi como capas o, mejor, mientras que él se va desdibujando para luego rearmarse de otra manera en ese tránsito. Solo cabría insistir en que la exploración del personaje y narrador de su propia conciencia y el contacto con distintas clases sociales (vinculadas al problema racial también) van de la mano, al menos en un primer momento: la metáfora geológica permite unir las dimensiones subjetiva y social.

En esta novela, el personaje principal sale de su ámbito cómodo y burgués (estrato seis colombiano), como todo heredero de empresario, hacia el peregrinaje en ámbitos sociales marginados y pobres, para culminar en el encuentro con la comunidad negra del Pacífico colombiano, una de las más pobres de todo el país. Hacia el final, la identificación del personaje con esa comunidad (la de su nana perdida) es explícita: "Y yo pienso rabioso, casi mascullo que no nos mata nadie, no nos pueden matar, no pueden, por mucho que intenten acabar con nosotros. A nosotros nos protege el diablo. A nosotros no nos mata ni el putas” (Cárdenas, 2013, p. 200).

Por su parte, en Lo que no aprendi, García Robayo relata dos historias (que parecen ser una) vinculadas por medio de dos partes. La primera parte cuenta la historia de Catalina, un personaje que narra episodios de su infancia en Cartagena de Indias en 1991, año de la Constitución Política actual de Colombia. Tales episodios están relacionados con su padre y un misterio que lo rodea debido a cierto ocultismo, además de contar con escenas ambiguas en torno al despertar sexual con Aníbal, un personaje adulto, posiblemente abusador. También encontramos escenas cotidianas de las vacaciones de Catalina con sus dos hermanas y su hermano menor, su madre y Mery, la empleada del servicio. La segunda historia es la de una narradora que pareciera ser la misma Margarita García Robayo en una versión autobiográfica. Cuenta el momento de su vida adulta en Buenos Aires en el que se entera de la muerte de su padre y decide escribir una novela relacionada con su familia. Elige un nombre para su protagonista, que será el de Catalina, de manera que esta segunda parte parece una suerte de texto metaficcional sobre la escritura de la primera. El asunto es que ni la 
narradora ni el personaje principal parecen ser las mismas en las dos partes; de ahí que hablemos, por el momento, de dos historias dentro de una misma novela.

Por otra parte, el título alude explícitamente a la novela misma. Como le dice la madre a la narradora adulta al final de la novela, "si no te gustan mis recuerdos, empieza a juntar los tuyos; y si tampoco te gustan esos, cámbialos, y así; es lo que hacemos todos. Le contesté, todavía llorando: yo no sé hacer eso. Y ella: entonces aprende" (García Robayo, 2014, p. 182). La novela sería entonces la historia de aquello que no se aprendió, es decir, a juntar o recrear recuerdos, en este caso, de la infancia y de ese momento tan crucial del país (por la Constituyente, pero también por la violencia y la presión en la política de los carteles del narcotráfico de Medellín y de Cali).

Ahora bien, el aprendizaje del manejo de los recuerdos emerge explícitamente en la segunda parte como un tema de la escritura, sin que la narración de la primera historia corresponda del todo con ese intento de escritura terapéutica sobre la infancia y el recuerdo. En cualquier caso, la lectura aislada de las partes generaría dos historias autónomas, casi como relatos separados; sin embargo, su lectura conjunta como una misma novela hace que la lectura de las dos partes se haga más compleja: no parece tratarse solo de un relato sobre la infancia en la Cartagena de los años noventa, en el primer caso, ni tampoco de las confesiones autobiográficas de una escritora adulta expatriada en Buenos Aires, en la segunda. La estructura de esta novela implica una lectura de conjunto, de modo que las dos historias o versiones chocan y se contradicen en diversos puntos: de ahí el gesto relevante de leerla como una novela y no como dos historias autónomas. Se trata de un texto que se construye a partir de esa yuxtaposición entre dos historias que trabajan sobre un mismo referente, pero que chocan y se contradicen, para subrayar lo que no se aprende por medio del relato.

Por su lado, en La ruidosa marcha de los mudos, Juan Álvarez trabaja un género literario que parecería denostado hoy en día: la novela histórica. Se trata de un texto que recrea de manera singular varios episodios de la declaración de Independencia en el Virreinato de la Nueva Granada, en 1810, y de lo que después se conoció como la "patria boba", es decir, el periodo hasta 1815 que termina con el sitio de Cartagena y el inicio de la sangrienta reconquista (o Régimen del Terror) a cargo de Pablo Mori1lo (conocido como el Pacificador), quien llega a Bogotá en 1816.

La novela construye episodios que van desde 1808 a 1816 en torno a un personaje mudo y de la clase popular, José María Caballero Llanos. A raíz del diario 
histórico del José María Caballero real, Dias de la Independencia. En La Patria Boba, y de la ficcionalización de las páginas arrancadas acerca de la declaración de la Independencia, Álvarez narra, en diez capítulos, episodios de la vida de este personaje negociante, mudo y ayudante de los criollos de la declaración de 1810. Esa vida ficcional de quien no tiene voz está involucrada con los criollos poderosos que participaron en el proceso de Independencia y a la vez con la chichería de su familia, en especial de su madre y de su hermana adoptiva, la india Nicolasa. Es así que un personaje funciona como una bisagra entre los criollos históricos con poder - como Camilo Torres y Francisco José de Caldas-, o quienes pensaron en una revuelta social real - como José María Carbonell, conocido como “el Chispero de la Revolución”- y personajes populares que aparecen recreados en el ámbito de la chichería, lugar de encuentro popular en la época.

El título de la novela alude a la mudez del personaje, pero al mismo tiempo al trabajo con una novela histórica más cercana a personajes populares que a una imagen mitificada y solemne de los próceres de la Independencia en Colombia; es decir, más próxima al ruido de los humildes. Progresivamente, desde la mirada del personaje mudo, Álvarez va avanzando hacia la decepción ante los próceres y la evaluación de esa historia desde la perspectiva de personajes populares y comunes. Como lo plantea el narrador hacia el final, a propósito de la decepción frente a los criollos,

Esa noche borracha, después de conocer detalles, Caballero regresa abatido a la casa de los suyos, con el disfraz de fe a medio poner y la sensación de que esas humillaciones sometidas, de que esas súplicas actuadas por los otrora líderes criollos, señores nobles a los que de cerca había servido y vocales del bando por el que había arriesgado, eran un asco imposible (Álvarez, 2015, p. 207).

Hasta aquí la presentación de la historia, la estructura y el tono de las tres novelas. Como vemos, se trata de temas distintos: una búsqueda aparentemente íntima y personal que termina con un encuentro en la selva con comunidades negras, en Los estratos; una reconstrucción de recuerdos personales ficcionalizados a partir de la imagen misteriosa y ausente del padre, en un marco general de Cartagena en la época de la Constituyente en los años noventa en Colombia, en Lo que no aprendi; y una ficcionalización de episodios de la "patria boba" en el siglo XIX a partir de un personaje de la clase popular mudo, en La ruidosa marcha de los mudos. Ahora bien, ¿̇en qué rasgos podemos ver la tensión entre experimentación y representación en estas tres novelas contemporáneas? 


\section{Formas de experimentación y representación}

Veamos algunos de los puntos que pueden dar luces al respecto. En el caso de la novela de Cárdenas, la representación emerge especialmente en la narración de condiciones sociales precisas durante el viaje del protagonista a través de los distintos estratos. A pesar de prescindir de los nombres propios de los personajes y los lugares, es identificable el referente al que se alude y el tipo de problemática que se describe. Además, el narrador evalúa con frecuencia la situación social de los personajes que va encontrando. Al llegar a lo que, deducimos, es Buenaventura, por ejemplo, menciona la música que se escucha desde afuera y dice:

La música. La maldita música es lo peor. Todas las canciones hablan de despecho, de amor no correspondido, de abandono y entonces me descubro pensando que esta gente habla de amor para no decir mierda, habla de amor para no decir hambre, habla de amor para no decir puta vida, mi casa se hunde, no tengo trabajo, no tengo en qué caerme muerto (Cárdenas, 2013, p. 155).

Como vemos, hay una representación de un lugar de enunciación particular de este personaje con respecto a la gente que lo rodea. Su lugar es el de un hombre blanco, de clase acomodada que está en condiciones muy diferentes a las que va a encontrar en los personajes que habitan en Buenaventura y en la selva. Esto se reconoce en sus juicios y en la descripción que hace de los espacios y de los otros personajes. Hay una representación de ese lugar de enunciación sin el cual la crítica social de la novela pasaría inadvertida. Pero además de esa representación tanto del lugar de enunciación del narrador como de las condiciones sociales de los distintos personajes — desde los machotes empresarios hasta la comunidad negra-, hay tres formas experimentales que nos parecen relevantes.

En primer lugar, está la desarticulación de un relato aparentemente intimista al inicio que se va rearticulando progresivamente en una experiencia colectiva de la desigualdad social. En otras palabras, en lugar del relato de la depresión de un burgués citadino y el derrumbe de su subjetividad, tenemos el acercamiento progresivo a una experiencia comunitaria, después de la quiebra económica y la ruptura social que supone su separación.

En segundo lugar, tenemos un uso de voces distintas al cerrar cada parte, voces que hibridan ideolectos del Pacífico colombiano, imitando la oralidad, con mensajes viejos de prensa. También hay unos capítulos oníricos que mantienen la inconclusión del relato. Aunque en la interpretación de Quesada (2017), “La destacada presencia 
de lo onírico contribuye, igualmente, a que el lector tenga una cierta sensación de asfixia ante ciertos pasajes inextricables que tienen, sin embargo, una fortísima carga ideológica” (p. 173), no consideramos que lo onírico esté exclusivamente vinculado a lo ideológico o, si lo está, su expresión no es discursiva ni aleccionante. Desde nuestro punto de vista, esas voces mantienen cierta apertura de la narración, puesto que son codas, pero no conclusiones, desenlaces o explicaciones, y ahí está su carácter experimental. Como lo dirá Cárdenas en otro texto a raíz de una experiencia pedagógica posterior en el Pacífico colombiano, "El río no se calla nunca. No se puede hacer callar al río” (Cárdenas y Álvarez, 2019, p. 129). Lo onírico tampoco se calla, pero no culmina en una explicación discursiva e ideológica, sino que entra a abrir ramas del relato principal, pero que no se cierran ni se concluyen, sino que confluyen (como en la imagen del río). Esa confluencia hace parte de la exploración de las formas asociadas a esos estratos subjetivos. En otras palabras, lo onírico puede ser ideológico también, sin que se presente en la novela en una versión reducida a lo discursivo y abiertamente pedagógico: es otra voz y otra forma literaria que se explora tanto en su cara delirante como ideológica.

Por último, está el uso desviado de dos géneros: el policial, cuando el detective indio parece apoyar la búsqueda de la nana, y el de novela psicológica, al encontrar la tumba de la que posiblemente fue la nana perdida. En esta medida, ni se resuelve la intriga de la "investigación" ni el encuentro con el hijo de la nana resuelve ningún trauma o la culpa de clase del protagonista. El cierre de estos asuntos se da solo en el encuentro con la comunidad, pero no como resolución o explicación: simplemente como un final que interrumpe el relato de la culpa individual.

En el caso de García Robayo, la representación es visible en el uso de referentes históricos concretos de 1991 y de la cultura popular de la época. Ahora bien, esos eventos parecen ser un marco accesorio de la historia de Catalina, no el centro de la narración. En este caso, es el relato familiar el que va a ocupar un espacio central. Sin embargo, en él también es clara la función referencial de ciertos comentarios clasistas de la madre del personaje, como en este episodio en el que leemos su discurso directo: "Es la última vez que te lo advierto: como te vuelva a encontrar oyendo conversaciones ajenas te reviento a gaznatazos. No soporto más esa bendita manía, pareces una muchacha del servicio"(García Robayo, 2014, p. 72). Aquí es evidente también la representación de cierto lugar de enunciación (un lugar clasista en la costa Caribe), 
en este caso familiar y visto desde la infancia, de una señora de clase acomodada que se refiere despectivamente a la clase social de las empleadas domésticas por considerarlas inferiores y de hábitos indeseables (como si el chisme fuese exclusivo de una clase social determinada o de un oficio particular). Hasta aquí los rasgos evidentes de la representación social e histórica.

Ahora bien, quisiéramos destacar dos rasgos de experimentación formal en esta novela. El primero y el más evidente es el desequilibrio y la asimetría de las dos partes y de las dos historias (tanto en extensión como en su narradora). Esta estructura hace que la novela desestabilice dos cosas: el supuesto relato autobiográfico de la primera parte y el supuesto relato metaficcional de la segunda. Al que creemos que es un relato autobiográfico sobre la infancia en la primera parte, se le atribuye el carácter de mera ficción que solo recrea materiales autobiográficos; al que pensamos que es el relato autobiográfico sobre cómo se escribió el primer relato, se le añade el componente metaficcional que pone en duda la responsabilidad de la enunciación (tanto del primer relato como del segundo). Es decir, al estar juntas esas dos partes, lo que se interpretaría como el relato de infancia dentro la novela y la explicación de cómo se escribió ese relato terminan por dejar indeterminada la función de uno y otro relato. Esto deja la novela en un estado de indeterminación con respecto a una lectura de las dos partes.

En esta medida, es una experimentación que violenta tanto la autobiografía como la metaficción, y lo hace dejando abierta la opción de cómo leer el texto en su conjunto; de cómo leer Lo que no aprendi como una novela. Esta estructura hace también que quede clausurada la posibilidad de una explicación: es una novela que no busca explicar ni sus episodios ni su construcción y que, incluso cuando lo intenta — como en el episodio del abuso sexual—, mantiene eludida la explicación. Este nos parece un rasgo experimental relevante, puesto que no es frecuente en textos que se enmarcan en contextos históricos y sociales tan definidos.

El segundo rasgo es el movimiento de la narradora en la primera parte. A veces narra de manera simultánea a los hechos (como si fuera Catalina niña presenciando eventos), y otras desde un momento de enunciación posterior a los eventos narrados (como si fuera una Catalina adulta). Técnicamente, a veces la narradora es homodiegética (simulando la niñez dentro del universo narrado) y a veces heterodiegética (simulando la distancia de la adultez). Ninguna de las dos coincide con la narradora de la segunda parte, de modo que este rasgo refuerza el cuestionamiento de la unidad de una sola historia y un 
solo relato. Es una novela que se construye a partir de esas inestabilidades en una historia de infancia en principio simple, inestabilidades que nos parecen destacables porque evitan formular una explicación. Precisamente no se aprende, porque no se puede explicar.

Por su parte, en Juan Álvarez tenemos que el rasgo de representación más fuerte tiene que ver con la necesidad de retomar y cuestionar la imagen que se tiene sobre la historia de la Independencia. El texto usa fechas concretas, nombres de personajes históricos y explicaciones del contexto, incluso da cuenta de las condiciones miserables en las que se encontraba el Virreinato de la Nueva Granada hacia 1808. Todos estos son recursos para la representación entendida como referencialidad histórica y social. Por otro lado, la novela expone dos rasgos experimentales que entran a dialogar con esa representación histórica. Primero está la elección - atípica en la novela histórica clásica, aunque quizá menos en la novelas históricas más recientes)— de la perspectiva de los humildes. Toda la novela, si bien gira en torno a episodios conocidos, está construida desde las tensiones entre el pueblo mestizo, los indígenas, las mujeres y los criollos, más que desde una versión maniquea entre los americanos y los españoles. El carácter experimental se ve particularmente en la elección de la mudez como rasgo central para recrear esas tensiones, mudez que se articula con el cuaderno de notas de Caballero Llanos. Si bien este personaje no puede hablar, sí observa y registra lo que ocurre con la marcha de los humildes, marcha que finalmente permite que haya existido esa revuelta de 1810. La reconstrucción de espacios populares como la chichería y de reclamos como los de la india Nicolasa por la promesa incumplida de matrimonio son, en este sentido, fundamentales, puesto que no han sido el centro de las recreaciones históricas. La trama se teje desde un ambiente popular cuya historia no ha tenido tanta difusión y que se construye por medio de la mudez, pero también con la posibilidad de la escritura.

El segundo rasgo es el tipo de lenguaje que construye el narrador: se trata de un registro informal que hibrida léxico y formas de la época con vocablos contemporáneos. Esta hibridación es constante y la podemos ver en pasajes como el siguiente a propósito de la madre de Caballero Llanos, cuando este se inicia como comerciante:

[...] lo reprendió por confundirla en la crianza de otro, que de tanto guerrerito le había salido era pícaro hijueputa. Preguntó por qué. Lloró sus varios no sé. Se acongojó y rezó y finalmente rogó, como lo había hecho siempre de la mano de su marido Mariano, por el buen camino y la distancia con los ladrones eternos de aquel reino malparido (Álvarez, 2015, p. 33). 
Este lenguaje híbrido entre lo formal y lo soez, y entre un léxico contemporáneo informal y otro de la época de la Colonia, hace que este narrador tenga un ideolecto propio muy marcado y humorístico en muchos casos. Este es un rasgo experimental que lo aleja inmediatamente de la objetividad de un narrador tradicional de novela histórica. Más que la ficción de una objetividad del narrador, tenemos acceso a la ficción de la sinceridad de sus tonos. Como vemos, incluso en este texto más ceñido a un género específico, como sería la novela histórica, las formas experimentales entran siempre a tensionar un relato que representa una historia y unas condiciones sociales determinadas.

\section{Cierre}

Después de este breve recorrido por tres textos colombianos contemporáneos, nos parece relevante insistir en que la representación, entendida como una forma referencial de narrar consensuada y comprensible por todos, es necesaria para hacer inteligibles los problemas que abordan estas novelas. No estamos ante poéticas que defiendan o trabajen desde juegos llamados posmodernistas, desde la experimentación entendida solo como la dimensión lúdica de la comunicación verbal —o como una suerte de solipsismo en la escritura - ni desde la hipernarrativización entendida como la acumulación y yuxtaposición de múltiples historias y anécdotas que se superponen como en películas de acción. Se trata de textos cuyos referentes sociales e históricos (e ideológicos, si se quiere) son reconocibles, y ese rasgo es uno de sus valores literarios. Quizá, ante este tipo de novelas, sea relevante que en un futuro la crítica indague por el funcionamiento de la referencialidad en la construcción de textos comunicables —en una dimensión más cotidiana que literaria-, sin que esto implique una reivindicación de que cualquier historia es literaria ni que todo texto literario deba explicitar sus referentes históricos, ideológicos o políticos para ser valorado literariamente.

Ahora bien, lo que usualmente llamamos representación se mantiene con frecuencia en un orden de cosas y en maneras de nombrar esas cosas que están muy ligados al estado de una situación determinada y a sus formas discursivas. Lo interesante de esta narrativa colombiana contemporánea es que incrusta formas experimentales para que podamos pensar y percibir de maneras distintas y no en los mismos términos en los que una situación se ha pensado, ignorado o reiterado históricamente (sea 
la sociedad colombiana contemporánea y su clasismo y racismo determinantes, sea el momento histórico de los años noventa o el proceso de Independencia). Esas formas de experimentación implican salir de los códigos recurrentes y comunes para pensar la historia y el presente de nuestras tradiciones.

En medio de este giro hacia lo referencial que anunciábamos al inicio, es un buen momento para recordar esa política de las formas literarias que ha explorado Rancière (2010) y el diálogo entre representación y experimentación que, creemos, le es indisociable. De acuerdo con Álvarez en su manifiesto pedagógico acerca de la experiencia en el Pacífico colombiano con la maestría en Escritura Creativa del Instituto Caro y Cuervo, "Intervenir desde el relato no es, no puede ser, escribir historias sobre el agua dulce para que los ciudadanos 'caigan en cuenta' de la finitud del recurso. Intervenir desde el relato tiene que ser construir la experiencia de la sed" (Cárdenas y Álvarez, 2019, p. 131). Tal creación de experiencias que aún no han tenido lugar (del todo) pasa por la experimentación con las formas y su relación tensa con la dimensión referencial del lenguaje. Estos textos son literarios y a la vez políticos no solo porque sus referentes sean políticos, históricos o sociales, sino porque en sus formas se construyen maneras distintas de experimentar y pensar en esos referentes, como reclamaba ya Walter Benjamin en 1934. Formas que desacomodan el lugar común sobre la historia, la sociedad y la política colombianas, además de la cultura y la literatura como expresiones asépticas frente a sus propios referentes. Esta incomodidad que aún produce el cruce entre experimentación y representación nos parece fundamental hoy en la tradición colombiana, especialmente en un momento en que nuestro epígrafe - de una novela de 1975- parece escandalosamente vigente en la Colombia de 2021.

\section{Referencias bibliográficas}

Álvarez, J. (2015). La ruidosa marcha de los mudos. Bogotá: Seix Barral.

Ángel, A. (1975). Estaba la pájara pinta sentada en el verde limón. Bogotá: Ediciones B.

Auerbach, E. (1950). Mimesis. La representación de la realidad en la cuttura occidental. México, D. F.: Fondo de Cultura Económica.

Becerra Grande, E. (2015).De la crítica latinoamericanista: el corto viaje contra sí misma. Delaware Review of Latin American Studies 15 (3). Recuperado de https://udspace.udel.edu/handle/19716/19756 Benjamin, W. (2009). El autor como productor. En Obras (Libro II, vol. 2, pp. 297-315). Madrid: Abada Editores. 
Cárdenas, J. (2013). Los estratos. Cáceres: Periférica.

Cárdenas, J.y Álvarez, J. (2019). Construir la experiencia de la sed (aproximaciones pedagógicas y políticas a la escritura creativa). La Palabra 34, pp. 123-132.

García Robayo, M. (2014). Lo que no aprendí. Barcelona: Malpaso.

Grützmacher, L. (2006). Las trampas del concepto "la nueva novela histórica"; y de la retórica de la historia postoficial. Acta poética 27 (1), pp. 141-167.

Gumbrecht, H. U. (2004). Production of Presence. What Meaning Cannot Convey. Stanford: Stanford University Press.

MacKay, M. (2011). The Cambridge Introduction to the Novel. Cambridge: Cambridge University Press.

Negrete Sandoval,J.É. (2015). Tradición autobiográfica y autoficción en la literatura hispanoamericana contemporánea. De Raíz Diversa 2 (3), pp. 221-242.

Ortiz, M. P. (2014). "La violencia en Colombia es una de las mil formas de la desigualdad”. Entrevista con Juan Cárdenas. El Tiempo (27 de junio 27). Recuperado de https://www.eltiempo.com/ archivo/documento/CMS-14179295

Quesada, C. (2017). Juan Cárdenas y la otra tradición. En V. Capote Daz y E. Ángel. Escribiendo la nación, habitando España (pp. 167-186). Madrid y Frankfurt: Iberoamericana-Vervuert.

Rancière, J. (2010). Politica de la literatura. Buenos Aires: Libros del Zorzal.

Tynianov, I. (1969). Destruction, parodie. Change 2, pp. 67-76. 